\title{
Diseño, Implementación y Evaluación de un Programa de Prevención de la Violencia en el Noviazgo
}

\author{
Design, Implementation and Evaluation of a Dating Violence Prevention Program
}

\author{
Ana María Póo \\ $\&$ \\ M. Beatriz Vizcarra \\ Universidad de La Frontera, Chile
}

(Rec: 5 de Octubre de 2010 / Acep: 8 de Agosto de 2011)

\begin{abstract}
Resumen
El presente artículo describe el diseño, implementación y evaluación del programa "Construyendo una relación de pareja saludable", dirigido a jóvenes de educación superior. El programa tiene como propósito prevenir la violencia de pareja en jóvenes a través del desarrollo de conocimientos, actitudes y habilidades, que les permitan la construcción de una relación de pareja basada en la igualdad y el respeto mutuo. El programa compuesto por 5 módulos y 14 sesiones semanales, se implementó como asignatura electiva para estudiantes de la Universidad de La Frontera. Los resultados de la evaluación indican que el diseño es apropiado al perfil de los usuarios, la implementación muestra adecuación de los contenidos y de la metodología de enseñanzaaprendizaje. La evaluación de los resultados de aprendizajes señala un aumento de los conocimientos y del rechazo a la violencia, los cuales se mantienen 6 meses después de terminado el programa.
\end{abstract}

Palabras clave: Programa educativo, prevención de la violencia de pareja, evaluación de programas, jóvenes de educación superior.

\begin{abstract}
This paper describes the design, implementación and evaluation of the program "Building a healthy relationship," for higher education students. The program aims to prevent dating violence through the development of knowledge, attitudes and skills that enable them to build a relationship based on equality and mutual respect. The program consists of 5 modules and 14 weekly sessions, and was implemented as an elective course for students of the Universidad de La Frontera (Chile).

The evaluation results indicate that the design is appropriate to the users' profile, the implementation assessment shows adequacy of contents and teaching methodology. The evaluation of students learning outcome revealed a significant increase in knowledge about dating violence, communication and nonviolent conflicto resolutions strategies and moderate increase rejection of violence, which remained 6 months after the end of the program.
\end{abstract}

Key words: Educational program, dating violence prevention, program evaluation,

higher education students.

* Correspondencia: Ana María Póo, Departamento de Psicología, Universidad de La Frontera, Casilla 54-D Temuco, Chile. E-mail: ampoo@ ufro.cl Esta investigación fue financiada por el Consejo Nacional de Ciencia y Tecnología Proyecto Fondecyt 1080394. 


\section{Introducción}

La violencia en el noviazgo o "dating violence" como se conoce en los países anglosajones, se define como abuso físico, emocional y sexual en una relación romántica estable en que no existe vínculo legal ni cohabitación (Ruiz \& Fawcett, 1999). Los estudios internacionales y nacionales sobre este tema demuestran que afecta un número significativo de jóvenes (Straus, 2004). En Chile una encuesta realizada por el Instituto Nacional de la Juventud en el año 2006, indicó que 13,4\% de los jóvenes reconoce haber sufrido violencia psicológica y 7,6\% violencia física, no existiendo mayor diferencia entre hombres y mujeres (Instituto Nacional de la Juventud, 2001). Por otra parte Vizcarra \& Póo (2011), en un estudio realizado en la región de la Araucanía, señalan que un número significativo de estudiantes (57\%) refiere haber sufrido algún episodio de violencia psicológica y $26 \%$ de violencia física leve de parte de su pareja. A su vez, $32 \%$ reconoce haber ejercido violencia psicológica y $8,1 \%$ violencia física, alguna vez en la vida.

Con respecto al impacto de la violencia en los jóvenes, distintos estudios han descritos la presencia de trastornos depresivos, deterioro de la autoestima, de la confianza y del sentimiento de seguridad, sentimientos de culpa, aislamiento, bajo rendimiento académico y mayor riesgo de consumo abusivo de substancias (O’Keefe, Brockopp \& Chew, 1986; Póo \& Vizcarra, 2008; Singer, Anglin, Song \& Lunghofer, 1995). Las consecuencias para quienes ejercen violencia son: riesgo de ruptura de la relación, sentimientos de vergüenza, rechazo y condena social, así como el riesgo de repetir el modelo de interacción violenta en futuras relaciones (Glass, Freland, Campbell, Yonas, Sharp \& Kub, 2003).

Una de las formas de intervención que han demostrado ser más eficaces para enfrentar este problema, ha sido la implementación de programas educativos, los cuales se han desarrollado principalmente en Estados Unidos y Canadá. Algunos de ellos son Viraj (Lavoie, Vezina, Piche \& Boivin, 1995), Youth Relations (Wolfe et al., 1996) y Safe Dates (Foshee, Bauman, Arriaga, Helms, Koch \& Linder, 1998). Este último, en un estudio de seguimiento realizado 4 años después del término del programa, mostró una disminución de 56 a 92\% de violencia física y sexual severa, además de incrementar las habilidades de comunicación, manejo de la ira y disminución de los estereotipos en los participantes (Foshee, Bauman, Ennett, Linder, Benefield \& Suchindran, 2004).

El éxito de los programas educativos se relaciona, por una parte, con un diseño riguroso de objetivos, contenidos, metodología, material didáctico y técnicas de enseñanza, las cuales deben ser consistentes con las características de la población destinataria (Cabrera, 2000). Por otra parte la evaluación de los programas de prevención de la violencia dirigido a jóvenes muestran que los más exitosos son aquellos que están insertos en el currículo, tienen continuidad en el tiempo, cuentan con un número suficiente de sesiones y se orientan al desarrollo de conocimientos, actitudes y habilidades (Cornelius \& Resseguie, 2007).

La ausencia de programas educativos orientados a la prevención de la violencia de pareja en jóvenes en nuestro país, impulsó a las autoras a desarrollar el programa "Construyendo una Relación de Pareja Saludable". Este programa recoge tanto las experiencias internacionales como los resultados de las investigaciones de las autoras (Foshee et al., 1998; Muñoz, 2009; Póo \& Vizcarra, 2008).

\section{Descripción del Programa}

El programa Construyendo una Relación de Pareja Saludable es un aporte a la prevención de la violencia y al desarrollo de relaciones de pareja saludables, destinado a estudiantes de educación superior. El diseño de un programa para este grupo objetivo, se fundamenta en que en esta etapa de la vida se construyen las bases de las relaciones de pareja más definitivas, por lo que la intervención en este momento, puede determinar la calidad de la relación futura. Además los jóvenes como futuros profesionales pueden constituirse en agentes de cambio que promuevan una actitud de respeto a la mujer y rechazo a las relaciones abusivas en los diferentes contextos laborales en los que se inserten.

El propósito de este programa es entregar conocimientos, desarrollar habilidades y formar actitudes favorecedoras de una convivencia de pareja basada en la igualdad y el respeto mutuo. Construyendo una Relación de Pareja Saludable está diseñado como una herramienta de prevención tanto primaria como secundaria, ya que está dirigido a jóvenes que no presentan situaciones de abuso, jóvenes que están en riesgo o viviendo abuso y aquellos que podrían ejercerla, tanto hombres como mujeres, debido a que los estudios realizados señalan una prevalencia importante de violencia cruzada. El programa ha sido diseñado para ser implementado como actividad académica de tipo electivo y sus objetivos y contenidos se corresponden con los propósitos de formación de competencias de tipo genérico que la mayoría de las universidades chilenas han establecido como prioritarias en la formación profesional. Si bien el programa fue diseñado para jóvenes de enseñanza superior, puede ser adaptado para su utilización en estudiantes de enseñanza secundaria.

\section{Fundamentación Pedagógica}

El programa se fundamenta en el paradigma pedagógico del constructivismo (Piaget, 1970), y su concepción del sujeto como organismo activo que busca adaptarse a las resistencias de su ambiente mediante la construcción de esquemas de respuestas cada vez más sofisticados. Este paradigma se complementa para su aplicación en el ámbito 
pedagógico con los aportes de Vigotsky (1978), quien enfatiza la importancia de la interacción y la cooperación social como base para el aprendizaje, y el papel de la cultura como elemento mediatizador de los procesos de construcción del conocimiento (Denegri, 1996a,b). De acuerdo a Álvarez (1997), este modelo propicia que el estudiante se convierta en gestor de su propio aprendizaje a través de actividades que le permitan exponer e intercambiar ideas, opiniones y experiencias con sus compañeros, convirtiendo así el aula en un foro abierto a la reflexión y al debate. Por otra parte el aprendizaje requiere trabajar o procesar la información que recibimos, ya sea a través de una experiencia directa y concreta o bien a través de una experiencia abstracta. Sin embargo, para que estas experiencias se transformen en conocimiento, señala Kolb (1984), deben ser elaboradas a través de la reflexión sobre ellas o experimentando de forma activa la información recibida. A partir de lo anterior, en el presente programa se incluyen distintas actividades prácticas que permiten "aterrizar los aprendizajes conceptuales" y por otra parte se estimula la reflexión del estudiante tanto en aula como extra-aula, facilitando el registro de sus aprendizajes y reflexiones en un portafolio personal.

Considerando la base constructivista del programa, los objetivos pedagógicos se han diseñado equilibrando objetivos conceptuales, procedimentales y actitudinales. Los objetivos conceptuales se definen como aquellos orientados al conocimiento de hechos, conceptos y principios teóricos abstractos. Estos objetivos se han intencionado particularmente en el primer módulo, puesto que los participantes deben conocer las bases teóricas de la violencia de pareja, lo que les permitirá comprender la complejidad del fenómeno. Los objetivos procedimentales se definen como aquellos dirigidos al aprendizaje de procedimientos, destrezas, habilidades y estrategias dirigidas a la consecución de un objetivo. Estos se aprenden en el hacer, por lo que en cada módulo del programa se han diseñado actividades orientadas al desarrollo de habilidades que permitan construir una relación de pareja saludable. Los objetivos actitudinales se definen como aquellos orientados a la formación valórica, implican el desarrollo de actitudes, el aprendizaje de normas $\mathrm{y}$ formas de convivencia. Este tipo de objetivos se presenta transversalmente en todas las sesiones del programa y su propósito es intencionar que los participantes desarrollen una actitud de rechazo a la violencia en la pareja.

\section{Diseño}

El programa está organizado en 14 sesiones, de dos horas y media cada una, las que se desarrollan en modalidad de taller (ver anexo 1). Los contenidos se estructuran en 5 módulos: El Módulo I Bases teóricas de la violencia está constituido por 5 sesiones: La Sesión 1, Conceptualización y tipos de violencia en la pareja, tiene como propósito entregar a los participantes elementos teóricos que les permitan reconocer la violencia en sus distintas manifestaciones y desarrollar una actitud crítica frente a esta problemática. En esta sesión se facilita la constitución del grupo a través de dinámicas de presentación y conocimiento entre los participantes, y se establecen las normas de funcionamiento. La Sesión 2, denominada La violencia y las relaciones de poder en la pareja, tiene como objetivo describir la distribución histórica del poder en las relaciones intergénero, su relación con el surgimiento de los estereotipos de género, y el ejercicio de la violencia, favoreciendo en los estudiantes una actitud crítica frente a los modelos culturales que sustentan y legitiman la violencia, de pareja. La Sesión 3, Modelos explicativos de la violencia presenta los principales modelos teóricos que permiten comprender la interacción de los factores individuales, familiares, sociales y culturales asociados al surgimiento y mantención de la violencia de pareja. La Sesión 4, Características de la violencia de pareja en jóvenes, tiene como objetivo conocer las características específicas de la violencia en los jóvenes, fundamentada en los resultados de las investigaciones tanto internacionales como nacionales que muestran diferencias respecto de la violencia de pareja en población adulta. La Sesión 5, Recursos y estrategias para abordar la violencia, entrega a los participantes conocimientos sobre el marco legal de la violencia en Chile y los recursos disponibles tanto a nivel nacional como local para enfrentarla.

El Módulo II Relaciones de pareja saludables: La importancia de la comunicación se organiza en 3 sesiones. La Sesión 1, Definiendo relaciones de pareja saludable, tiene como objetivos reconocer las características de una relación de pareja satisfactoria y reflexionar acerca de la importancia del amor, el respeto mutuo basado en la igualdad, y la construcción de la intimidad. La Sesión 2, Comprendiendo las bases de la comunicación, tiene como propósito entregar a los participantes antecedentes teóricos que les permitan comprender la relevancia de la comunicación en las relaciones humanas, valorando la asertividad como base de una relación saludable. La sesión 3, Técnicas de comunicación asertiva, está orientada a entregar herramientas prácticas que les permitan desarrollar habilidades de comunicación asertiva como base de una relación saludable fundamentada en la equidad.

El Módulo III Autoconocimiento y expresión emocional se estructura en 3 sesiones. La Sesión 1, Conociéndonos, tiene como objetivo facilitar el autoconocimiento de modo que les permita reconocer características personales que puedan constituirse en un factor de riesgo de sufrir y/o ejercer violencia. La sesión 2, Identificación y expresión de sentimientos, se orienta a facilitar en los participantes el reconocimiento y la expresión adecuada de sus sentimientos, base para una comunicación eficaz y un manejo adecuado de conflictos en las relaciones de pareja. La Sesión 3 , Reconocimiento y manejo de la ira, tiene como objetivos que los participantes identifiquen situaciones que despiertan su ira, reconozcan las claves físicas y psicológicas que ésta 
genera, así como las estrategias que utilizan regularmente para reducirla desarrollando recursos para disminuir los niveles de tensión asociados.

El Módulo IV Resolviendo los conflictos de forma no violenta se compone de 2 sesiones. La Sesión 1, Bases teóricas del conflicto y estrategias de resolución, tiene como propósito mostrar a los participantes distintos tipos de conflicto, sus elementos constitutivos y las estrategias de resolución colaborativas y no colaborativas, favoreciendo una valoración positiva del uso de las estrategias no violentas. La Sesión 2, Habilidades de resolución no violenta de conflictos, está destinada a facilitar en los participantes el desarrollo de habilidades colaborativas para la resolución de conflictos y la valorización de este tipo de habilidades aplicadas a las relaciones de pareja. El Módulo V Integración está compuesto de una sesión, Compartiendo mis aprendizajes, y tiene como propósito facilitar la integración de los conocimientos, habilidades y valores en relación a la violencia de pareja desarrollados durante el programa educativo. Como objetivos adicionales se plantea la evaluación del programa y el cierre formal a través de una actividad social o recreativa.

\section{Implementación}

El Programa Construyendo una relación de pareja saludable se implementó como asignatura de carácter electivo dirigida a estudiantes de la Universidad de La Frontera entre marzo y julio de 2009, con una matrícula de 31 estudiantes y un nivel de asistencia superior al 70\%. Las sesiones fueron conducidas por las investigadoras, quienes estuvieron a cargo del desarrollo de las actividades. En la primera sesión se entregó a cada estudiante un portafolio que incluía los objetivos y las actividades de cada sesión junto con un disco compacto (CD) con las presentaciones audiovisuales de las sesiones y artículos relacionados con los contenidos. El portafolio tenía como objetivo que los estudiantes registraran sus aprendizajes y reflexiones, de modo que les permitiera evaluar su trabajo y tomar conciencia de sus logros.

\section{Evaluación del Programa}

Evaluación del Diseño

Una vez elaborado el programa se llevó a cabo una evaluación del diseño, con el fin de valorar su coherencia interna, es decir, la coherencia de los objetivos con el perfil de los participantes, la adecuación de las estrategias y la temporalización. Esta evaluación fue realizada por una profesional externa con formación académica en psicología y pedagogía y amplia experiencia en docencia en educación superior.

\section{Evaluación de la Implementación:}

Instrumento y Estrategias

Pauta de evaluación: Este instrumento se aplicó a los estudiantes al final de cada sesión y al término del programa para conocer su valoración respecto de los contenidos, metodología, material didáctico y monitores a través de una escala de 1 a 6 , considerando un puntaje de 1 como muy malo y 6 equivalente a excelente. Además la pauta incluía una evaluación cualitativa de indicadores ya señalados y una autoevaluación.

Grupo focal: tres semanas después de finalizado el programa se realizó un grupo focal con 5 participantes destinado a conocer la percepción respecto de éste, el nivel de satisfacción y las sugerencias para su mejoramiento.

\section{Evaluación de resultados de aprendizaje}

Para la evaluación de los aprendizajes se utilizaron dos instrumentos, los cuales se aplicaron al inicio y al final del programa, tanto al grupo estudio compuesto por los 31 estudiantes que cursaron el programa, como al grupo control constituido por 34 estudiantes pareados en función de edad, sexo y carreras con el grupo estudio. Los instrumentos aplicados fueron:

Cuestionario de Conocimientos: este instrumento, construido por las autoras estaba compuesto por 35 ítems de respuesta objetiva que evaluaban los contenidos abordados en el programa.

Escala de actitudes: este instrumento, construido por las autoras, constaba de 12 ítems, con formato de respuesta tipo Likert de 5 opciones, el cual indagó el nivel de justificación de los estudiantes respecto del uso de la violencia en la pareja Se estimó una confiabilidad a través de la prueba alfa de Cronbach de 0,90.

\section{Evaluación de seguimiento:}

Entrevistas focalizadas: Con el fin de evaluar la permanencia de los aprendizajes, ayudantes de investigación entrevistaron a seis participantes, seis meses después de terminado el programa. La pauta de entrevista estaba compuesta por 8 preguntas, enfocadas a evaluar las competencias logradas a través del programa.

\section{Resguardos éticos}

Cada participante, tanto del grupo control como del grupo estudio, firmó un consentimiento donde se informó de los objetivos de la evaluación, enfatizando el tratamiento confidencial de los datos, los cuales se utilizarían solo con fines de investigación. 

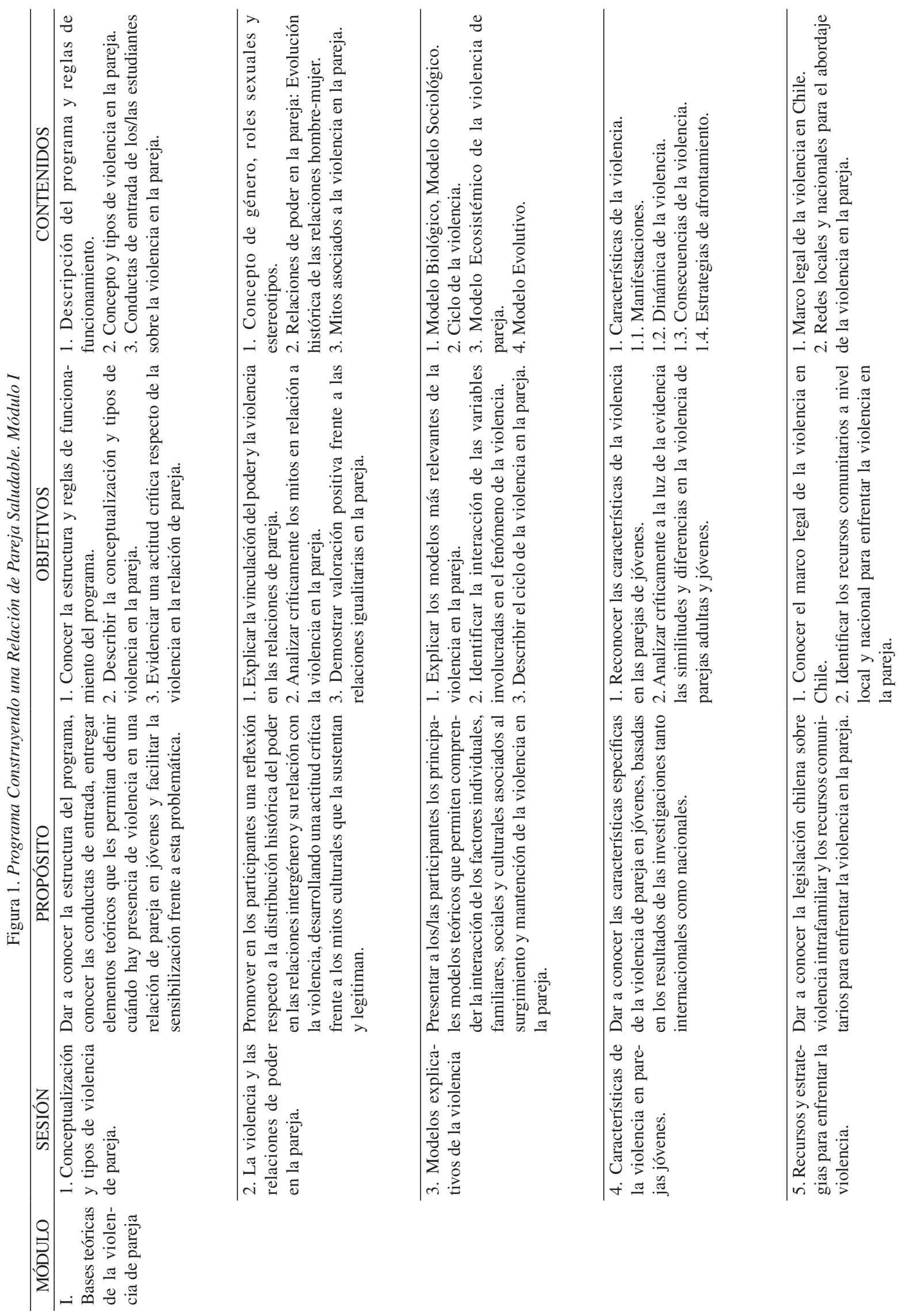


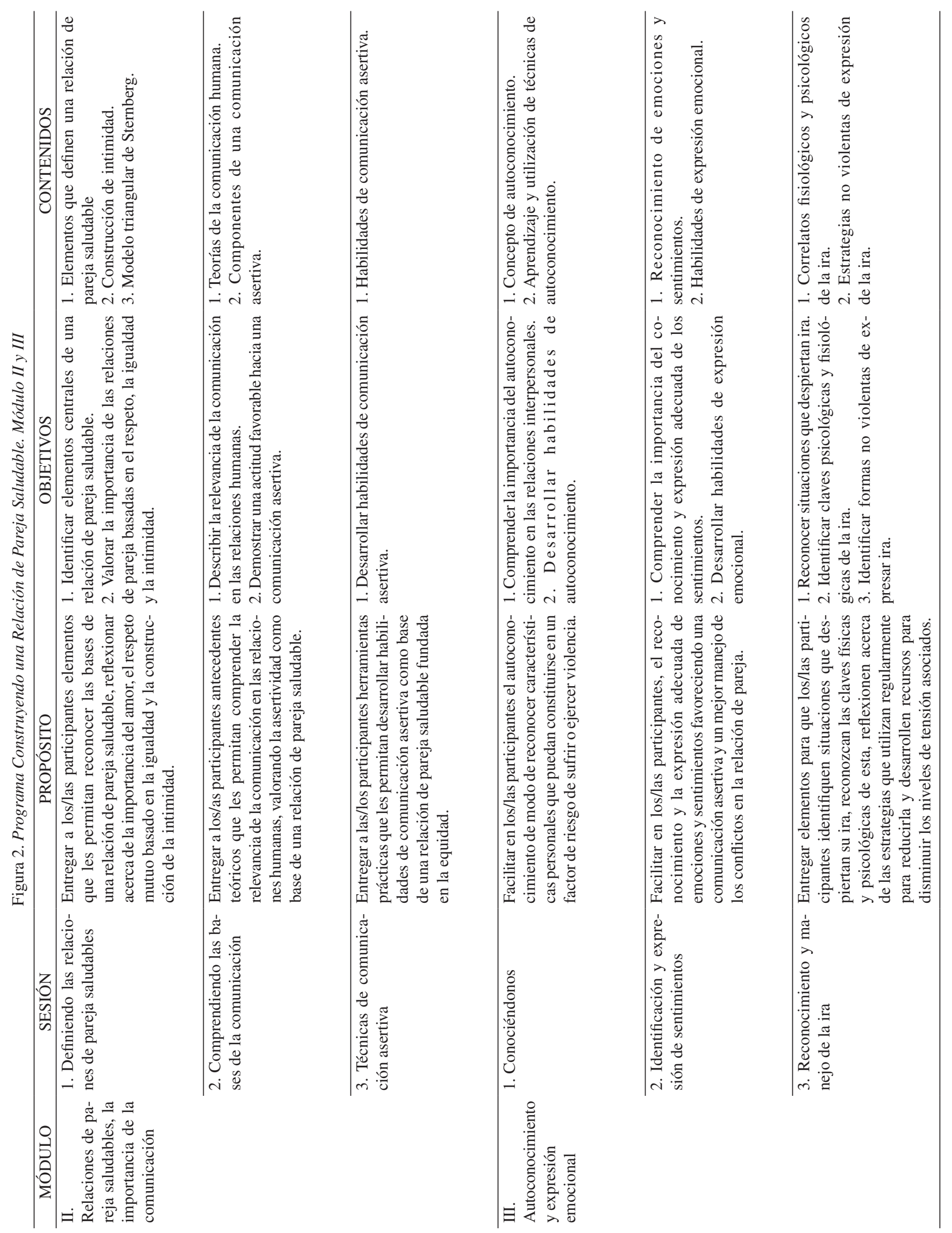



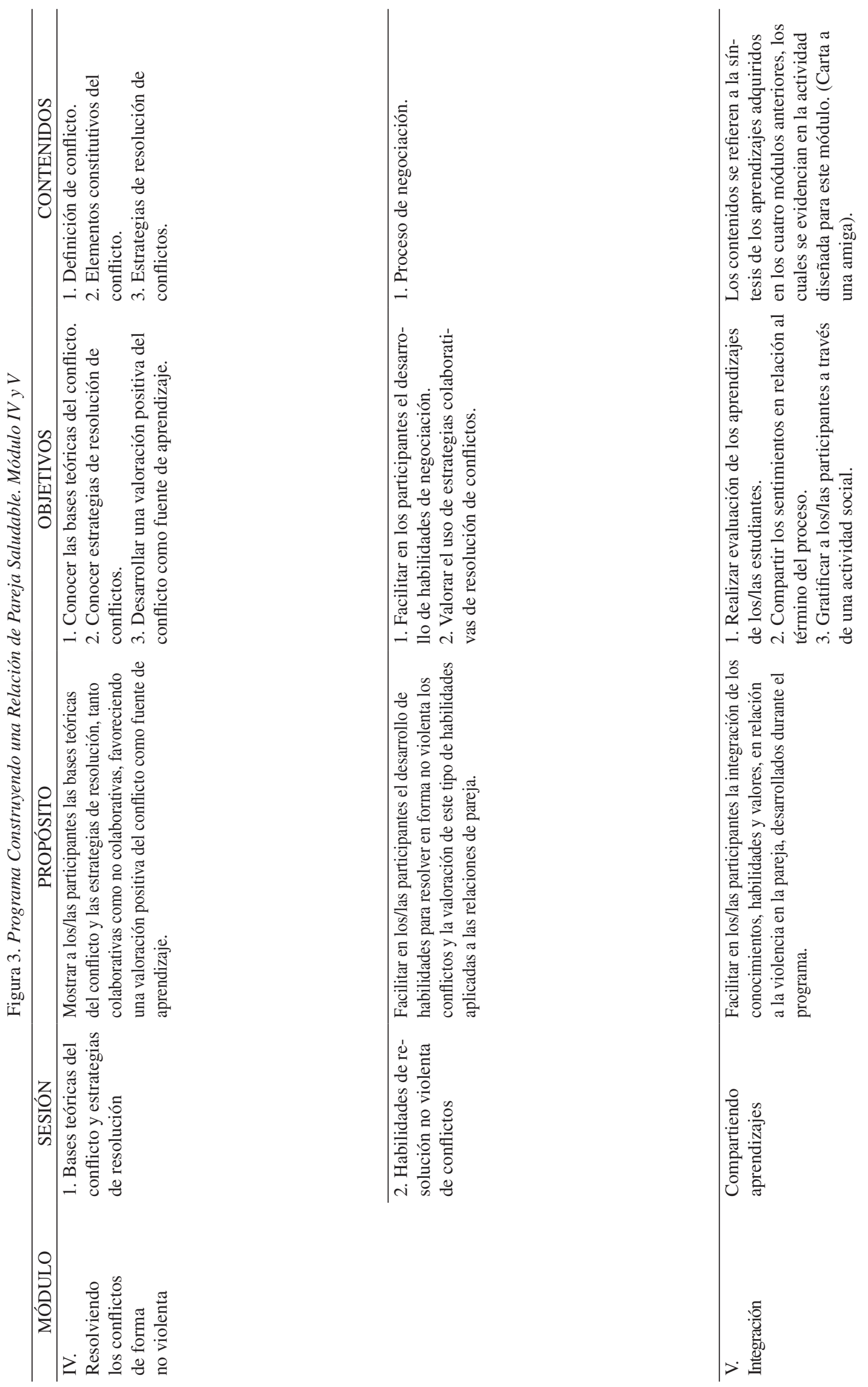


\section{Resultados}

\section{Evaluación del diseño del Programa}

La evaluación del diseño realizada por profesional experto concluyó que el programa presentaba coherencia entre objetivos, contenidos y metodología, las cuales respondían al perfil de los usuarios. Se sugirieron cambios menores a nivel de redacción de objetivos e instrucciones y tiempos asignados a las actividades.

\section{Evaluación de la implementación del Programa}

Pauta de evaluación: La información cuantitativa entregada por los estudiantes a través de la pauta de evaluación se presenta en la tabla 1 .

Con respecto a la evaluación cualitativa contenida en la pauta, los estudiantes hacen referencia a fortalezas y aspectos a mejorar en relación a tres dimensiones.

\section{Fortalezas}

Contenidos: Estos fueron considerados relevantes y útiles para la vida personal y profesional: "muy aplicables y de gran utilidad para resolver conflictos de pareja u otras interacciones, con el fin de alcanzar un alto grado de bienestar personal y otorgar estabilidad a la relación de pareja", "los ejemplos que se dieron fueron muy cercanos a nuestra realidad de pareja, lo que me llevó a autoevaluarme de algunas actitudes en mi relación".

Metodología: Fue valorada positivamente, destacando la coherencia entre los aspectos teóricos y las actividades prácticas en cada sesión y la coherencia entre las distintas sesiones: "durante el módulo la forma de abordar los contenidos y las actividades realizadas fueron muy buenas, ya que las actividades eran muy pertinentes a lo que se mostraba en la teoría y permitieron poner en práctica y discutir cada contenido visto, además de hacer más amena la clase".

Por otra parte, los estudiantes destacaron el aprendizaje significativo logrado a través de diversas estrategias pedagógicas: "muy bueno el trabajo de relajación", "me gustó el aprendizaje que se puede lograr a través de juegos, podemos recordar gracias a estos por más tiempo el sentido de las sesiones", "compartir experiencias en una ronda final supuso integrar de buena forma los aportes de los estudiantes", "los trabajos en grupo sirven bastante para hablar con personas

Tabla 1. Valoración cuantitativa. Programa Construyendo una relación de pareja saludable

\begin{tabular}{|c|c|c|c|}
\hline Criterios & $\mathrm{M}$ & Criterios & M \\
\hline Contenidos & 5.5 & Material didáctico & 5.4 \\
\hline Calidad & 5.4 & Calidad & 5.5 \\
\hline Profundidad & 5.2 & Diseño & 5.4 \\
\hline Actualidad & 5.8 & Pertinencia & 5.4 \\
\hline Coherencia temática & 5.7 & Facilita aprendizaje & 5.4 \\
\hline Pertinencia & 5.6 & Monitores & 5.6 \\
\hline Metodología de Trabajo & 5.5 & Capacidad para trasmitir conocimiento & 5.6 \\
\hline Claridad y precisión objetivos & 5.4 & Preparación y actualización & 5.6 \\
\hline Calidad técnica & 5.4 & Complementariedad & 5.6 \\
\hline Integración aportes estudiantes & 5.4 & Experiencia y aporte profesional & 5.6 \\
\hline Calidad y coherencia entre actividades & 5.6 & Capacidad de motivar & 5.1 \\
\hline Aprovechamiento del tiempo & 5.3 & Acoger e inspirar confianza & 5.1 \\
\hline Motivación generada por las actividades & 4.8 & Consistencia & 5.6 \\
\hline Contribución al aprendizaje & 5.4 & Apertura y flexibilidad & 5.6 \\
\hline Portafolio del estudiante & 5.4 & Asistencia y puntualidad & 5.7 \\
\hline Calidad & 5.6 & Autoevalaución & 4.6 \\
\hline Diseño & 5.5 & He sido éticamente responsable & 4.8 \\
\hline Claridad instrucciones & 5.3 & Me he relacionado respetuosamente & 4.8 \\
\hline Pertinencia & 5.3 & Riguroso en mis opiniones & 4.7 \\
\hline \multirow[t]{3}{*}{ Facilita aprendizaje autónomo } & 5.3 & He aceptado el error y la diferencia & 4.6 \\
\hline & & Asistencia puntualidad y permanencia & 4.1 \\
\hline & & He sido responsable en mis tareas & 4.0 \\
\hline
\end{tabular}

Escala: 1 a 6 
de otras carreras y aunque no siempre hay acuerdo, se sale más fortalecido. Compartir las conclusiones con los otros grupos permite ver ideas diferentes a las tuyas, te aportan".

Material didáctico: Los estudiantes destacaron la pertinencia del material usado, que facilitó la discusión y participación: "el material que se entrega en cada sesión facilita el aprendizaje, tanto grupal como individual, ya que es práctico, didáctico y de fácil entendimiento", "me pareció que la carta de Alejandra ${ }^{1}$ puede ser la realidad de cualquier joven de nuestra sociedad, por tanto, un hecho de toda actualidad", "fomenta la discusión y participación abriendo el tema al curso".

\section{Aspectos a mejorar}

Entre las sugerencias propuestas por los estudiantes destacaron: disminuir los tiempos de las presentaciones teóricas, y destinar mayor tiempo al trabajo interactivo: "me gustaría más tiempo para analizar y discutir los temas", "los power eran muy largos y poco esquemáticos". Por otra parte sugieren dedicar más tiempo a temas relacionados con estrategias que faciliten el autoconocimiento y el desarrollo de relaciones de pareja satisfactorias: "faltó más sesiones del módulo de auto conocimiento, y menos del primer módulo". En relación a la metodología, sugieren que la "carta de Alejandra" sea aplicada no sólo al final del programa, para evaluar resultados de aprendizaje, sino también al inicio, para evaluar conductas de entrada.

Grupo focal: Coincidente con la pauta de evaluación, la información obtenida se organizó en tres dimensiones temáticas: contenidos, metodología y material didáctico.

Contenidos: Los participantes valoraron principalmente los temas relacionados con autoconocimiento, herramientas de comunicación asertiva y estrategias de resolución de conflictos: "los temas igual los encontré súper buenos, porque mas allá de ver la violencia en sí, a mí personalmente me enseñó a comunicarme asertivamente, a entender al otro y a verse uno".

\section{Metodología}

Los estudiantes valoraron las actividades prácticas, como juegos de roles y análisis de casos: "a mí me tocó participar en varios e igual uno aprende con eso, porque se mete en el papel y se da cuenta de la situación que está enfrentando". En relación al tamaño de los grupos de trabajo, señalaron que la participación se incrementó en grupos pequeños, lo cual facilitó el aprendizaje. Por otra parte destacaron la formación de grupos al azar, ya que favoreció el intercambio de experiencias con estudiantes de otras carreras: "en el grupo chico uno habla y los otros

Nota: la carta fue un recurso pedagógico para evaluar competencias para ayudar a sus pares que viven relaciones de violencia. Esta se aplicó en la última sesión. escuchan y como que es más cercano el ambiente, pero en el grupo grande es más difícil".

Material didáctico, destacaron que el material audiovisual resultó útil no sólo para la formación personal, sino también para la formación profesional: "a nosotras nos tocó un trabajo, en otra asignatura, de violencia en la mujer y las presentaciones nos sirvieron muchísimo".

Asimismo, el portafolio fue considerado de utilidad como guía de las sesiones: "a mí me sirvió como guía, al comienzo salían todos los temas, los módulos, entonces eso igual me ayudó a ver cómo iba a ser la estructura”.

Por último, como sugerencias proponen incorporar al comienzo del programa más actividades que generen confianza intragrupal con el objeto de favorecer la participación, realizar más trabajo de terreno como asistir al tribunal de familia para ver un caso de violencia y destinar más tiempo para el desarrollo de algunas actividades.

\section{Evaluación de los aprendizajes}

Cuestionario de conocimientos: Los resultados se expresaron en una escala de 1 a 7 . Si bien tanto el grupo estudio como el grupo control mostraron un incremento de sus conocimientos entre la medición pre y post programa, el incremento en el grupo estudio fue altamente significativo (tabla 2).

Escala de actitudes: Los resultados mostraron un aumento estadísticamente significativo en el rechazo a la violencia en el grupo estudio comparado con el grupo control (tabla 3).

\section{Evaluación de seguimiento}

En cuanto a los aprendizajes significativos, la mayoría de los entrevistados señalaron que antes del programa sólo consideraban las manifestaciones de violencia física. Actualmente reconocen que son capaces de identificar otros tipos de violencia, como violencia psicológica y sexual, reconocen además que la violencia se presenta a través de ciclos. Algunos entrevistados reportaron identificar variables que permiten predecir conductas violentas “... "he visto gente que no sabe controlar sus impulsos, no saben cómo decir a veces las cosas... de repente se puede llegar a los golpes".

En cuanto a los recursos personales desarrollados a través del programa, los entrevistados señalaron asertividad, empatía y comunicación: "yo aprendí que uno tiene que ser capaz de saber expresar lo que está sintiendo, y que uno siempre tiene que negociar"... "la comunicación es fundamental, y también el ponerse en el lugar del otro y ser empático, pensar en cómo reaccionaría si me estuviera sucediendo lo mismo".

Otros entrevistados destacaron la relevancia del autoconocimiento, vinculado a un proceso reflexivo: "principalmente el proceso cognitivo y reflexivo que significa el 
Tabla 2. Incremento en conocimientos en grupo estudio y grupo control.

\begin{tabular}{lccccc}
\hline & \multicolumn{2}{c}{ Inicio del Programa } & \multicolumn{2}{c}{ Final del Programa } & \multicolumn{2}{c}{$p$} \\
& M & DS & M & DS & $0.0001^{* * *}$ \\
Grupo estudio & 2.7 & 0.58 & 6.1 & 0.99 & $0.015^{*}$ \\
Grupo control & 2.9 & 0.61 & 3.1 & 0.68 & 0.1 \\
\hline
\end{tabular}

$*=\mathrm{p}<0.05 ; * *=\mathrm{p}<0.01 ; * * *=\mathrm{p}<0.001$

Tabla 3. Incremento en el rechazo a la violencia en grupo estudio y grupo control

\begin{tabular}{|c|c|c|c|c|c|}
\hline & \multicolumn{2}{|c|}{ Inicio del programa } & \multicolumn{2}{|c|}{ Final del programa } & \multirow[b]{2}{*}{$p$} \\
\hline & M & DS & M & DS & \\
\hline Grupo estudio & 16.8 & 6.71 & 20.6 & 4.15 & $0.007 * *$ \\
\hline Grupo control & 15.7 & 6.77 & 16.2 & 7.72 & 0.561 \\
\hline
\end{tabular}

$*=\mathrm{p}<0.05 ; * *=\mathrm{p}<0.01 ; * * *=\mathrm{p}<0.001$

autoconocimiento, el cual me ha ayudado bastante en mi formación profesional".

Algunos estudiantes plantearon que han llevado a la práctica conocimientos adquiridos en el programa, tomando un rol protagónico de apoyo y acompañamiento a sus pares: "antes del electivo jamás hubiese pensado en intervenir en una situación así, al menos que hayan sido personas desconocidas, en cambio ahora me siento con una responsabilidad de que si puedo ayudar en algo debo hacerlo"... "me ayudó a comprender más, soy capaz de ver otros factores que están influyendo en la situación".

Uno de los entrevistados aludió también a una permanente actitud y conducta proactiva en torno a este tipo de problemática: "yo vengo de una familia en la que se dio la violencia... siempre me metí y yo creo que viéndolo allá afuera, en la calle, si me tocara verlo lo haría". Otro estudiante describió un cambio en cuanto a la relevancia que le otorga en el presente a factores protectores como la autoestima: "valorarte como persona y quererte... quererte mucho", así como también factores de riesgo: "levantarse todas las mañanas y andar todo el día como con lata... uno permite que otras personas te hablen igual".

Así también destacaron como aprendizaje significativo, un mayor análisis ante situaciones consideradas como violentas, incluyendo en esta comprensión las influencias del entorno y la relación con las personas involucradas: “el asunto de resolución de conflictos, si bien no me acuerdo de los pasos uno por uno, sí tengo una comprensión de ayudar a ponerlos en práctica"... "comprender qué lo está llevando a comportarse de esa forma, qué cosas le ha pasado, qué está viviendo, por qué tiene esa frustración o esa rabia, todas esas cosas".

\section{Discusión}

Con respecto a la evaluación del programa, cabe destacar la positiva apreciación de los estudiantes, considerando que en la evaluación cuantitativa el promedio se acercó al puntaje máximo en todas las dimensiones.

Respecto de los contenidos la valorización de la actualización y coherencia temática, da cuenta de un diseño adecuadamente fundamentado en resultados de investigaciones internacionales y estudios locales realizados en población similar a la del grupo objetivo al cual se orientó el programa (Cabrera 2000). En relación a la pertinencia de contenidos como autoconocimiento, comunicación asertiva y resolución no violenta de conflictos, la valoración de los estudiantes reafirma lo propuesto por González \& Santana (2001), quienes recomiendan que los programas se orienten a adquirir información sobre relaciones saludables, un factor importante a la hora de modificar las conductas de riesgo y desarrollar habilidades que favorezcan relaciones basadas en el respeto y la equidad (Wolfe \& Feiring, 2000). La metodología de orientación constructivista y el material didáctico diseñado para el desarrollo de las actividades es valorado en la medida que les permitió concretizar contenidos teóricos por medio del dialogo, la reflexión y aplicación práctica. En particular, los estudiantes destacaron el uso del portafolio como facilitador del aprendizaje autónomo.

La evaluación de los estudiantes respecto de su desempeño particularmente referido a responsabilidad en el cumplimiento de tareas, asistencia y puntualidad, es más crítica comparada con las otras dimensiones de la pauta de evaluación. Este grado de autocrítica permite suponer que la apreciación respecto del programa no está sesgada por la deseabilidad social.

La evaluación de los resultados de aprendizajes muestra claramente el incremento en la apropiación de los contenidos por parte de los estudiantes del grupo estudio, a diferencia del grupo control, donde el cambio fue marginal. Es importante relevar que estos conocimientos facilitan en los estudiantes una visión integral del problema incluyendo el reconocimiento de formas más sutiles de violencia, 
sus diversas manifestaciones, las variables que facilitan el surgimiento de ésta y las consecuencias asociadas.

La evaluación de seguimiento muestra que los aprendizajes adquiridos a través del programa tienen permanencia en el tiempo, destacando la internalización de responsabilidad social por parte de los estudiantes frente a la violencia en la pareja. Esto se evidencia en la disposición a intervenir cuando se enfrentan a situaciones de violencia que afectan particularmente a sus pares.

En relación al cambio de actitudes, es importante destacar la diferencia significativa entre el grupo estudio y control, lo que demuestra que el programa tiene impacto en este nivel. Esto es particularmente significativo, considerando que al igual que en otros estudios (Avery-Leaf, Cascardi, O'Leary \& Cano, 1997), el piso fue alto, ya que en la evaluación inicial, $73 \%$ de los estudiantes, tanto del grupo estudio como del grupo control, contestaron que la violencia nunca se justificaba.

Por último es importante destacar que las sugerencias propuestas por los estudiantes con respecto al programa se relacionan con aspectos formales como adecuación de los tiempos e incorporación de actividades que aumenten la interacción grupal, lo que no significa cambios sustanciales en el diseño del programa.

En relación a las limitaciones del presente estudio, es necesario mencionar el bajo número de participantes entrevistados en la evaluación de seguimiento, ya que sólo fue posible acceder a una muestra reducida.

Finalmente cabe destacar que el programa "Construyendo una relación de pareja saludable", constituye un aporte pionero a la prevención de la violencia destinado a jóvenes de educación superior en Chile. La evaluación del diseño, la implementación y los resultados de aprendizaje, avalan la utilidad del programa como un instrumento de prevención.

\section{Referencias}

Álvarez, M. (1997). Nuevas aproximaciones del Proceso de EnseñanzaAprendizaje. Una mirada desde la Misión Institucional. Revista Calidad de la Educación. Publicación del Consejo Superior de Educación. Santiago de Chile.

Avery-Leaf,S., Cascardi, M., O'Leary, K. \& Cano, A. (1997). Efficacy of a dating violence prevention program on attitudes justifying aggression. Journal of Adolescent Health, 21, 11-17.

Cabrera, F. (2000). Evaluación de la Formación. Madrid: Síntesis.

Cornelius, T. \& Resseguie, N. (2007). Primary and secondary prevention programs for dating violence: A review of the literature. Aggression and Violent Behavior, 12, 364-375.
Denegri, M. (1996 a). Las ideas de Piaget y Vigotsky. Santiago, Chile: La Salle.

Denegri, M. (1996 b). Desarrollo y aprendizaje: Las ideas de Piaget y Vigotsky en el Aula. Santiago, Chile: La Salle.

Foshee, V.A., Bauman, K.E., Arriaga, X.B., Helms, R.W., Koch, G.G. \& Linder, G.F. (1998). An evaluation of safe dates: An adolescent dating violence prevention program. American Journal of Public Health, $88,45-50$.

Foshee, V., Bauman, K., Ennett, S., Linder, F., Benefield, T. \& Suchindran, C. (2004). Assessing the long-term effects of the safe dates program and a booster in preventing and reducing adolescent dating violence victimization and perpetration. American Journal of Public Health, 94, 619-624.

Glass N., Freland, N., Campbell, J., Yonas, M., Sharp, P. \& Kub, J. (2003). Adolescent dating violence: Prevalence, risk factors, health out-comes, and implications for clinical practice. Journal of Obstetrics, Gynecologic, Neonatal Nursing, 32, 227-237.

González, R. \& Santana, J. (2001). Violencia en parejas jóvenes. Madrid: Pirámide.

Instituto Nacional de la Juventud. (2006). Informe resumido de resultados de la tercera encuesta nacional de juventud. Tomo 1. Santiago. Chile.

Kolb, D. A. (1984). Experiential Learning: Experience as the source of learning and development. New Jersey: Prentice-Hall.

Lavoie, F., Vezina, L., Piche, C. \& Boivin, M. (1995). Evaluation of a prevention program for violence in teen dating relationships. Journal of Interpersonal Violence, 10, 516-524.

Muñoz, G. (2009). Magnitud y características de la violencia de pareja en los estudiantes de la facultad de medicina de la Universidad Mayor: Una propuesta de trabajo pedagógico. Tesis para optar al grado académico de Magíster en Pedagogía Universitaria. Temuco.

O'Keefe, N.K., Brockopp, K. \& Chew, E. (1986). Teen dating violence. Social Work, 31, 465-468.

Piaget, J. ( 1970). Science of education and psychology of the child. Nueva York: Orion.

Póo, A. M. \& Vizcarra, M. B. (2008). Violencia de pareja en jóvenes universitarios. Terapia psicológica, 26, 81-88.

Ruiz, G. \& Fawcett, G. (1999). Rostros y máscaras de la violencia en el noviazgo. México: IDEAME.

Singer, M. I., Anglin, T. M., Song, L.Y. \& Lunghofer, L. ( 1995). Adolescent's exposure to violence and associated symptoms of psychological trauma. Journal of the American Medical Association, 273, 477-482.

Strauss, M. A. (2004) Prevalence of violence against dating partner by male and female university students worldwide. Violence Against Women, 7, 790-811.

Vigotsky, L. (1978). Mind in society: The development of higher psychological Cambridge: Harvard University Press.

Vizcarra, M. B. \& Póo, A. M. (2011). Violencia de pareja en estudiantes universitarios del sur de Chile. Universitas Psychologica, 10, 89-98.

Wolfe, D., Wekerle, R., Gough, R., Reitzel-Jaffe, D., Grasley, C., Pittman, A., Lefevbre, L. \& Stumf, J. (1996). The youth relationships manual. California: Sage Publications.

Wolfe, D.A. \& Feiring, C. (2000). Dating violence through the lens of adolescent romantic relationships. Child Maltreatment, 5, 59-3. 